\title{
Ion Beam Surface Modification of GaN Films for High Efficient Light Emitting Diodes
}

\begin{abstract}
G.M. WU $\mathrm{U}^{a, b, *}$, Y.S. $\operatorname{LIN}^{a}$ AND K.N. TU ${ }^{b}$
${ }^{a}$ Institute of Electro-Optical Engineering, Chang Gung University, Kweisan, Taoyuan 333, Taiwan R.O.C. ${ }^{b}$ Dept. of Materials Science and Engineering, University of California, Los Angeles, CA 90095, USA

Focused gallium $(\mathrm{Ga})$ ion beam technology has been proposed to modify the surface of GaN thin films. Due to the significant advancement in nitride semiconductors, the solid-state light emitting diodes will gradually replace fluorescent lamps in the next decade. However, further improvements in light extraction and power efficiency are still highly desired. GaN is limited by its high refractive index, with low light escape cone angle at about $24.6^{\circ}$. The external quantum efficiency is low due to the unwanted reflection and absorption. As the patterning technology scales down to the nanometer level, photonic crystal lattice in the visible light wavelength range can be achieved. Therefore, we improved the external efficiency by the new design of hexagonal photonic crystal lattice with air hole arrays in the diameter of $150 \mathrm{~nm}$ and the depth of $120 \mathrm{~nm}$. The Ga beam was accelerated at $30 \mathrm{kV}$ and the ion current was $100 \mathrm{pA}$. The plane wave expansion method along with the finite difference time domain was useful to investigate the quantum confinement. The nanopatterning by the focused ion beam could save time and processing step. In addition, we have successfully prepared blue InGaN/GaN samples with hexagonal period of $200 \mathrm{~nm}$. The device micro-photoluminescence results have demonstrated that the peak illumination intensity was improved by $30 \%$.
\end{abstract}

DOI: 10.12693/APhysPolA.123.884

PACS: 41.75.Ak, 81.65.-b, 42.70.Qs, 77.84.Bw

\section{Introduction}

Group III-nitride compound semiconductors, such as gallium nitride $(\mathrm{GaN})$, have been developed as important thin film materials for the successful commercialization of light emitting diodes (LED) in the green-to-blue spectral range. They have also paved the way for all-solid-state singlechip white illuminating devices to replace fluorescent lamps. There are always inevitable demands for the continued improvements in light extraction efficiency and lowered power consumption [1-3]. Although the internal quantum efficiency of the GaN-based LED can be as high as $98-100 \%$, only a small fraction of the emitted light can be intrinsically extracted. This is largely due to the interface refraction between the high index semiconductor materials and the ambient environment. The critical angle for the light extraction cone is as low as $24.6^{\circ}$ [4]. The light outside the extraction cone is thus repeatedly reflected and may become absorbed by the semiconductor thin films, metal electrodes and the oxide substrates.

Surface roughening by wet chemical etching provides a conventional means to reduce internal light reflection and to scatter more light outward. However, the uniformity has been difficult to control [5]. As the patterning technology scales down to the nanometer level, surface photonic crystal structure in the scale range of visible light wavelength can be achieved. Park et al. proposed an anodized aluminum oxide technique for nanopatterning transfer [6]. It could be used for large area patterning, but the pore arrangement was not very uniform. Kim

*corresponding author; e-mail: wu@mail.cgu.edu.tw et al. studied colloidal lithography and laser holographic lithography for GaN, and reported an output enhancement of $22 \%$ [7]. Yang et al. used indium tin oxide nanoparticle printing technique to improve light extraction efficiency by up to $28 \%$ at an injection current of $20 \mathrm{~mA}$ [8]. A more precise control of the surface nanopatterning could be developed by high energy focused gallium (Ga) ion beam technology. The technique provides one-step patterning and has been quite adaptive with complicated designs [9]. It saved time and processing steps in comparing different structures. A computer program could automatically carry out the entire patterning process.

In this study, we prepared InGaN/GaN semiconductor LEDs using the low-pressure metal-organic chemical vapor deposition system (MOCVD). The peak emission wavelength has been $468 \mathrm{~nm}$. The hexagonal photonic crystal lattices were produced by the focused gallium (Ga) ion beam using a SMI 3050 dual-beam nanotechnology workstation system. The nanopatterning technique required no photo mask. The various lattice design parameters were evaluated by the R-soft simulation tools to investigate the possible quantum confinement phenomena that could increase the light extraction efficiency, including the plane wave expansion (PWE) method along with the finite difference time domain (FDTD) method. The fundamental device photon extraction was then measured by a micro-photoluminescence (micro-PL) system using a $\mathrm{He}-\mathrm{Cd}(325 \mathrm{~nm})$ laser source.

\section{Experimental}

The epitaxial layers of GaN LEDs were grown on (0001) $\mathrm{Al}_{2} \mathrm{O}_{3}$ substrates by low-pressure MOCVD. 
During the growth, trimethylgallium (TMGa), trimethylindium (TMIn), and ammonia $\left(\mathrm{NH}_{3}\right)$ were used as the precursors of $\mathrm{Ga}$, In, and $\mathrm{N}$, respectively. The carrier gas was $\mathrm{H}_{2}$ and $\mathrm{N}_{2}$ for the growth of GaN and $\mathrm{In}_{0.2} \mathrm{Ga}_{0.8} \mathrm{~N}$, respectively. The GaN thin film of $50 \mathrm{~nm}$ in thickness was deposited as the buffer layer then undoped GaN layer $(2 \mu \mathrm{m})$ and $n$-type (Si-doped) GaN layer $(2 \mu \mathrm{m})$. The active multiple quantum well (MQW) layers were grown at $700-800^{\circ} \mathrm{C}$. They consisted of six periods of wells $(4 \mathrm{~nm})$ and barriers $(12 \mathrm{~nm})$. The final and top $p$-type (Mg-doped) GaN layer is $180 \mathrm{~nm}$ in thickness. The emission peak wavelength was designated at $468 \mathrm{~nm}$.

We prepared hexagonal photonic crystal (PC) lattice formed by air hole arrays created in the Mg-doped $p$-GaN layer (refractive index $\approx 2.4$ ). The bare wafers were dry-etched to create MESA for contact pads, and then processed by the focused ion beam (FIB) using the SMI 3050 dual-beam nanotechnology workstation system. Figure 1 shows the schematic illustration of the processing steps. The hexagonal air-hole array covered an area of $60 \times 60 \mu \mathrm{m}^{2}$, while each LED device had a lighting area of $65 \times 65 \mu \mathrm{m}^{2}$. The FIB acceleration voltage was $30 \mathrm{kV}$ and the ion beam current was $100 \mathrm{pA}$. The diameter of the air holes was $150 \mathrm{~nm}$ and the depth was about $120 \mathrm{~nm}$. The duration for the $\mathrm{Ga}$ ion beam to drill each hole was about $600 \mu \mathrm{s}$.

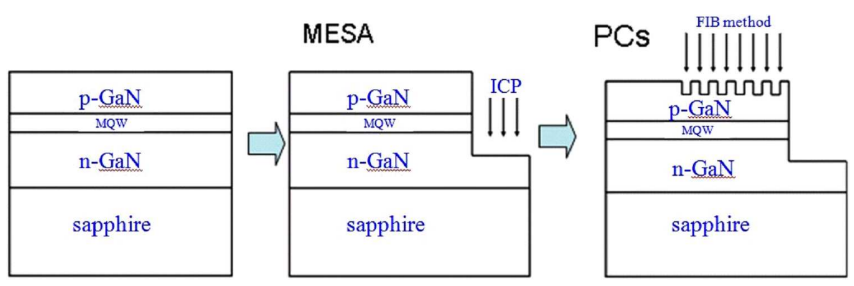

Fig. 1. Schematic illustration of the processing steps for the hexagonal photonic crystal lattice formed on GaN LED.

The various photonic crystal air-hole lattice parameters were studied by Rsoft's BandSOLVE using the plane wave expansion method to evaluate the band gaps and the band diagrams. The corresponding light wave propagation could be further investigated by the FullWAVE method. The air-hole array period ranged $200-800 \mathrm{~nm}$ in this study. The InGaN/GaN samples optical characteristics were measured by micro-PL (Jobin Yvon/Labram $\mathrm{HR}$ ) at room temperature using the $\mathrm{He}-\mathrm{Cd}(325 \mathrm{~nm})$ laser source.

\section{Results and discussion}

Table lists the various lattice period (a) constants and the corresponding photonic crystal simulation parameters used in the design study. The air hole radius $(r)$ was fixed at $75 \mathrm{~nm}$, and the LED emission wavelength $(\lambda)$ was $468 \mathrm{~nm}$. Figure $2 \mathrm{a}$ shows the band diagram calculated for the $p$-GaN substrate with refractive index of 2.4 and period of $200 \mathrm{~nm}$. The $r / a$ ratio is 0.375 and it has an air-filling factor $\approx 33 \%$. The TM band gaps are indicated by shaded bands in the range of $a / \lambda=0.33-0.44$, or $\lambda=455-606 \mathrm{~nm}$. The major emission wavelength of our sample devices has been within this wavelength range. On the other hand, the band diagram study indicated infrared $(\lambda=967-1071 \mathrm{~nm})$ band gaps for the sample with the period of $300 \mathrm{~nm}$. The LED sample with $a=400 \mathrm{~nm}$ exhibited no band gap and the band diagram is given in Fig. 2b for comparison. The other samples with larger period constants essentially suggested no band gap at all.

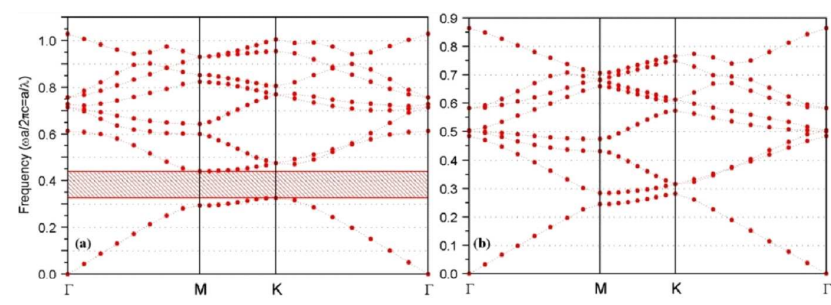

Fig. 2. TM band diagrams for $p$-GaN substrates with the refractive index 2.4 and the period of (a) $200 \mathrm{~nm}$ and (b) $400 \mathrm{~nm}$. The corresponding band gaps are indicated by shaded bands.

TABLE

Various lattice constants and corresponding photonic crystal simulation parameters used in the design study.

\begin{tabular}{c|c|c|c|c|c|c|c}
\hline \hline $\begin{array}{c}\text { Lattice } \\
\text { constant } \\
{[\mathrm{nm}]}\end{array}$ & 200 & 300 & 400 & 500 & 600 & 700 & 800 \\
\hline$r / a$ ratio & 0.375 & 0.25 & 0.188 & 0.155 & 0.125 & 0.107 & 0.094 \\
$a / \lambda$ ratio & 0.43 & 0.645 & 0.86 & 1.075 & 1.29 & 1.505 & 1.72 \\
$\begin{array}{c}\text { air-filling } \\
\text { factor [\%] }\end{array}$ & 33.13 & 14.73 & 8.28 & 5.3 & 3.68 & 2.72 & 2.13 \\
\hline
\end{tabular}

We further varied the air hole radius $(r)$ in the range of $20,40,60,75,80,100 \mathrm{~nm}$, while keeping the lattice period constant fixed at $200 \mathrm{~nm}$. The corresponding band diagrams (Figs. 2a and 3) exhibited band gaps for the LED samples with the radius of 60,75 , and $80 \mathrm{~nm}$. However, the corresponding $\lambda$ has been $555-689 \mathrm{~nm}$ for the $r=60 \mathrm{~nm}$ LED sample, which is not in the blue emission wavelength range of our LED wafers. The air-filling factor becomes too high when the hole radius $(r)$ is chosen at 80 or $100 \mathrm{~nm}$, which can make the FIB nanopatterning process very difficult and provide low yield. Therefore, we recommended the suitable radius of $75 \mathrm{~nm}$ for the FIB study.

Figure 4 displays the effects of the hexagonal lattice period constants on the light emission intensity by simulation. The period of $200 \mathrm{~nm}$ LED sample would demonstrate the highest light intensity. In addition, several other factors such as etching defects can contribute to nonradiative recombination, resulting loss in the form of heat. The air holes can provide some surface states, and capture negative electrons and positive holes.

Figure 5 shows the SEM micrographs of the FIB Ga ion drilled GaN samples. The hexagonal lattice con- 


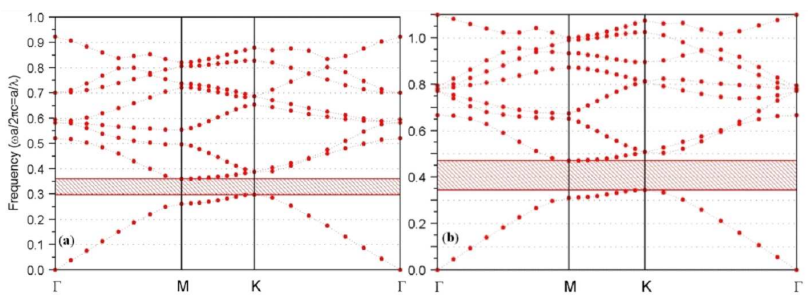

Fig. 3. The band diagrams exhibited corresponding band gaps for the LED samples with the radius of (a) $60 \mathrm{~nm}$ and (b) $80 \mathrm{~nm}$. The lattice period constant was fixed at $200 \mathrm{~nm}$.

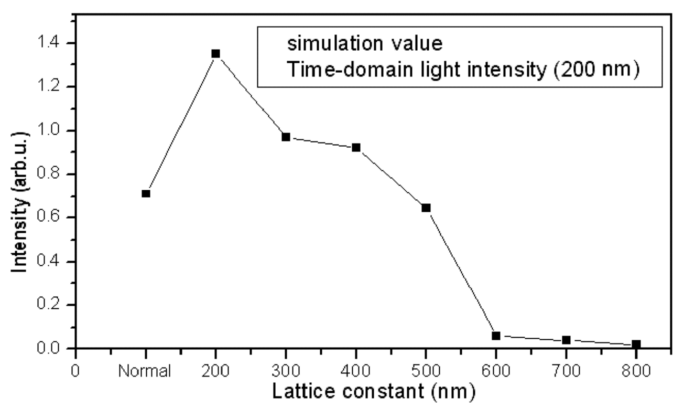

Fig. 4. Effects of hexagonal lattice period constants on the light emission intensity by FDTD simulation.

stant was $200 \mathrm{~nm}$. The air hole radius was $75 \mathrm{~nm}$ (150 nm in diameter), and the depth was $120 \mathrm{~nm}$. No metal (Pt) coating has been needed since the Mg-doped $p$-type GaN was conducting enough to spread the surface charges. The distribution is fairly uniform. We intended to drill deeper holes (about $200 \mathrm{~nm}$ ), but the drilling rate exponentially slowed down and that made deep hole drilling somehow impractical. The SEM micrographs of LED samples with the various hexagonal lattice constants of $300 \mathrm{~nm}, 400 \mathrm{~nm}$, and $500 \mathrm{~nm}$ are also provided in Fig. 6. The FIB processing technique would deliver adequate nanopatterning results as we designed. Further experiments could thus be carried out for the challenging industry.
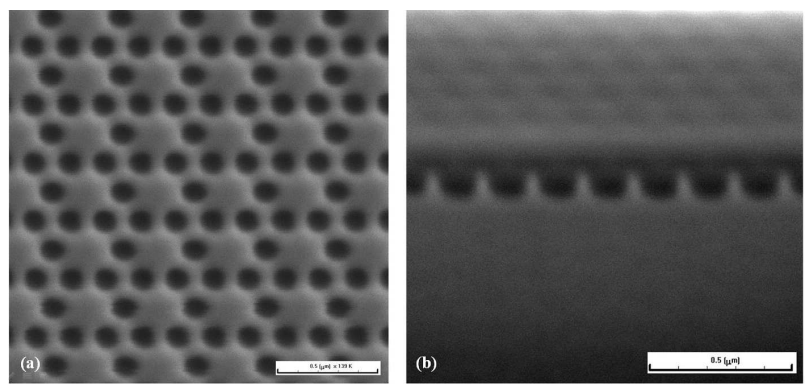

Fig. 5. SEM micrographs of the FIB Ga ion drilled GaN samples: (a) top view and (b) cross-section view. No metal coating has been needed.

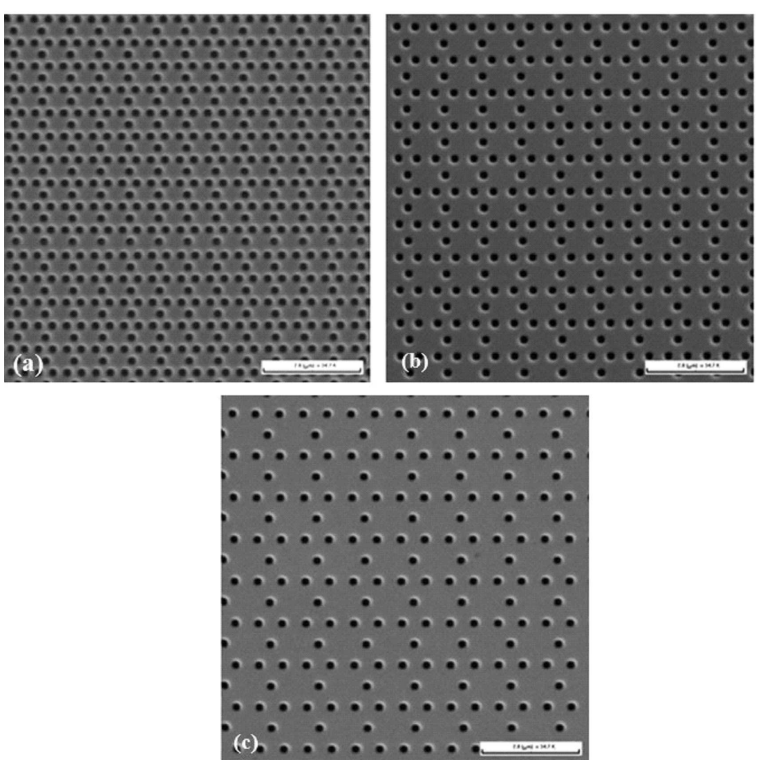

Fig. 6. SEM micrographs of the LED samples with hexagonal lattice constant of (a) $300 \mathrm{~nm}$, (b) $400 \mathrm{~nm}$, and (c) $500 \mathrm{~nm}$. The FIB processing technique delivered adequate nanopatterning results.

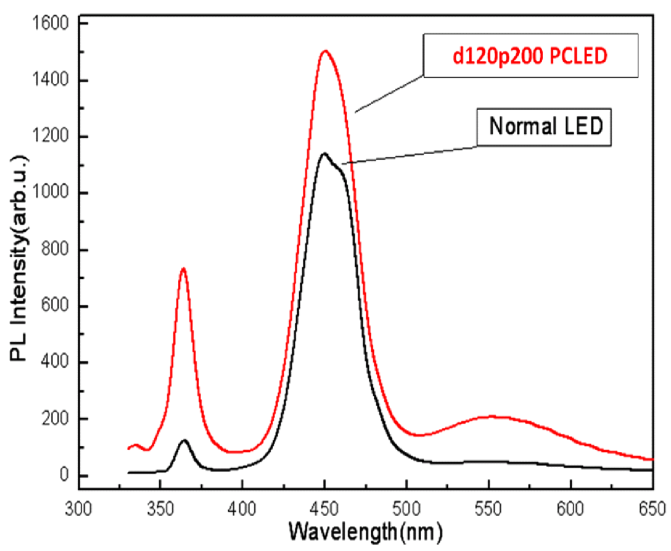

Fig. 7. Micro-PL intensity results of the InGaN/GaN samples with the FIB hexagonal photonic crystal lattices and normal samples (without the hexagonal lattice).

The FIB-drilled InGaN/GaN sample devices' photon extraction ability was measured by micro-PL using $325 \mathrm{~nm}$ laser source. Figure 7 displays the PL intensity results of the samples with the hexagonal lattice constant of $200 \mathrm{~nm}$ and the air hole diameter of $150 \mathrm{~nm}$ in the spectrum range of $350-650 \mathrm{~nm}$. The results of the samples without the hexagonal photonic crystal lattice (designated as normal LED) are also included for comparison. It has been clearly evidenced that the peak illumination intensity in the range of $460-470 \mathrm{~nm}$ had been improved by $30 \%$. The intrinsic illumination of GaN near $360 \mathrm{~nm}$ was also noted to increase by the hexagonal photonic crystal surface structures. The external quantum 
efficiency could thus be successfully improved by the photonic crystal lattices created by the FIB Ga beam surface modification. It will be very interesting and useful to further investigate the electrical excitation and discuss light emission at various wavelength ranges.

\section{Conclusions}

In the simulation study, we have found the ideal hexagonal photonic crystal lattice parameters for GaN: the period of $200 \mathrm{~nm}$ and radius of $75 \mathrm{~nm}$. The $r / a$ ratio was 0.375 and the air-filling factor was about $33 \%$. The results exhibited band gaps that were corresponding to the emission wavelength of $455-606 \mathrm{~nm}$. We further successfully prepared InGaN/GaN experimental devices with the ideal hexagonal photonic crystal lattice parameters using the accelerated Ga beam by the FIB dual-beam nanotechnology workstation system. The nanopatterned samples showed an improvement of $30 \%$ in the peak illumination intensity of $460-470 \mathrm{~nm}$ when compared with the counterpart samples without the FIB-patterned nanophotonic crystal lattice. Therefore, the light extraction and power efficiency can be further improved for industrial applications.

\section{Acknowledgments}

This work was supported by the National Science Council under research grants NSC99-2221-E182-001 and NSC100-2918-I-182-003.

\section{References}

[1] S. Watanabe, N. Yamada, M. Nagashima, Y. Ueki, C. Sasaki, Y. Yamada, T. Taguchi, K. Tadatomo, H. Okagawa, H. Kudo, Appl. Phys. Lett. 83, 4906 (2003).

[2] H. Kim, J. Cho, J.W. Lee, S. Yoon, H. Kim, C. Sone, Y. Park, T.Y. Seong, Appl. Phys. Lett. 90, 161110 (2007).

[3] G.M. Wu, Z.J. Cai, J.C. Wang, T.E. Nee, Surf. Coat. Technol. 203, 2674 (2009).

[4] T.X. Lee, K.F. Gao, W.T. Chien, C.C. Sun, Opt. Expr. 15, 667 (2007).

[5] S.I. Na, G.Y. Ha, D.S. Han, S.S. Kim, J.Y. Kim, J.H. Lim, D.J. Kim, K.I. Min, S.J. Park, IEEE Photon. Technol. Lett. 18, 1512 (2006).

[6] J. Park, J.K. Oh, K.W. Kwon, Y.H. Kim, S.S. Jo, J.K. Lee, S.W. Ryu, IEEE Photonics Technol. Lett. 20, 321 (2008).

[7] S.M. Kim, K.S. Kim, G.Y. Jung, J.H. Baek, H. Jeong, M.S. Jeong, J. Phys. D, Appl. Phys. 42, 152004 (2009).

[8] K.Y. Yang, S.C. Oh, J.Y. Cho, J. Electrochem. Soc. 157, H1067 (2010).

[9] G.M. Wu, B.H. Tsai, S.F. Kung, C.F. Wu, Acta Phys. Pol. A 120, 42 (2011). 